Sharif University of Technology
Scientia Iranica
SCIENTIA

\title{
New groundwater flow equation with its exact solution
}

\author{
A. Atangana* and C. Ünlü \\ a. Institute for Groundwater Studies, Faculty of Natural and Agricultural Sciences, University of the Free State 9300 Bloemfontein, \\ South Africa. \\ b. Department of Mathematics, Faculty of Sciences, University of Istanbul, 1293 Istanbul, Turkey.
}

Received 6 May 2015; received in revised form 21 July 2015; accepted 7 October 2015

\section{KEYWORDS}

New groundwater equation;

Beta-derivative;

Analytical methods;

Simulations.

\begin{abstract}
A new model of groundwater flowing within a confined aquifer was proposed using the concept of local derivative with fractional order. The derivative, used in this model, obeys all the properties of a local derivative, and has a fractional order. The new groundwater flow equation was solved analytically via three different analytical methods. The first method was the well-known method of separation of variables. The problem with this method is the introduction of the eigenvalue that does not have physical meaning. The second method was achieved using novel integral equation called Atangana-transform, and this method yields an exact solution. An alternative method based on the modified Boltzmann transformation also yields the exact solution. Some numerical simulations were done to express the efficiency of the model.
\end{abstract}

(C) 2016 Sharif University of Technology. All rights reserved.

\section{Introduction}

In the past decade, several researchers have devoted their attention to investigate the flow of groundwater within the geological formation called aquifer. The first mathematical equation describing this problem was proposed by Theis, however, we shall mention that this equation was borrowed from the heat model where the medium through which the head transfer is homogeneous [1-4]. The mathematical equation proposed by Theis uses the local derivative. However, in the last century, it was revealed in many branches of sciences that the derivative proposed by novel is not able to accurately describe some complex physical occurrences, for instance, this derivative cannot account for the variability of an aquifer. Anomalous diffusion processes in complex media can be well characterized by using fractional-order diffusion equation models [5-9]. The

\footnotetext{
*. Corresponding author. Tel.: +27 782948604;

Fax: +27 r95065268

E-mail addresses: abdonatangana@yahoo.fr (A. Atangana); unlucanan@hotmail.com (C. Ünlü)
}

time derivative term is corresponding to the long-time heavy-tailed decay and the spatial derivative for nonlocal diffusion. In 2006, Botha and Cloot proposed the model of groundwater flow using the Riemann-Liouville fractional order derivative [10]. Although the numerical simulation showed good agreement with experimental data, it is required to consider that the physical motive of and the physical meaning of this model cannot be explained. For instance, the initial condition used for their model does not have physical meaning because there is no fractional initial condition in nature. In addition, the derivative used in their study describes the flow in the global scale; therefore, one cannot describe the flow at the borehole, as it is local. In 2013, further studies using the Caputo derivative were done at the Institute for Groundwater Studies, South Africa, and the good news with the Caputo derivative is that it allows the use of the traditional initial condition, however, one cannot describe the flow at local scale [1114]. To take into account the variability of the aquifer together with the locality of the flow, we propose the use of the new derivative called beta-derivative. This derivative has been used in many fields, for instance, 
in epidemiology. Another concept of local derivative with fractional parameter was used in the following works [15-19]; however, this derivative cannot be used in groundwater flow problem because the derivative has been proposed to take care of fractal observations which are not found in an aquifer. In this study, we aim to propose a new model for the flow of groundwater within a confined aquifer using the beta derivative [20]. The structure of this paper is as follows: in Section 2, we shall present some useful properties of the betaderivative; in Section 3, we shall present the derivation of the new equation; in Section 4, shall we present the derivation of analytical solution using three analytical methods, and finally in Section 5, we intend to present numerical simulation with experimental data.

\section{Some information about beta-derivative}

Definition 1: Let $f$ be a function, such that, $f:[a$, $\infty) \rightarrow \mathbb{R}$. Then, the beta- derivative is defined as:

$$
{ }_{0}^{A} D_{x}^{\beta}(f(x))=\lim _{l \rightarrow 0} \frac{f\left(x+l\left(x+\frac{1}{\Gamma(\beta)}\right)^{1-\beta}\right)-f(x)}{l}
$$

for all $a \leq x, \beta \in(0,1]$. Then, if the limit of the above exists, $f$ is said to be beta-differentiable $[15,16]$.

It can be claimed that the above definition does not depend on the interval. If the function is differentiable, our definition at a point zero is different from zero.

Theorem 1. Assume that $g \neq 0$ and $f$ are two functions beta-differentiable with the $\beta \in(0,1]$, then the following relations can be satisfied [14]:

1. ${ }_{0}^{A} D_{x}^{\beta}(a f(x)+b g(x))=a_{0}^{A} D_{x}^{\beta}(f(x))+b_{0}^{A} D_{x}^{\beta}(g(x))$ all $a$ and $b$ are real number;

2. ${ }_{0}^{A} D_{x}^{\beta}(c)=0$ for $c$ any given constant;

3. ${ }_{0}^{A} D_{x}^{\beta}(f \cdot g(x))=f(x)_{0}^{A} D_{x}^{\beta}(g(x))+g(x)_{0}^{A} D_{x}^{\beta}(f(x))$;

4. ${ }_{0}^{A} D_{x}^{\beta}\left(\frac{f(x)}{g(x)}\right)=\frac{f(x)_{0}^{A} D_{x}^{\beta}(g(x))-g(x)_{0}^{A} D_{x}^{\beta}(f(x))}{g(x)^{2}}$.

The proofs of the above relations are the same as the one in $[15,16]$.

Theorem 2: Assume that $f:[a, \infty) \rightarrow \mathbb{R}$ be $a$ function such that $f$ is differentiable and also betadifferentiable. Let $g$ be a function defined in the range of $f$ and also differentiable, then we have the following rule:

$$
\begin{aligned}
{ }_{0}^{A} D_{x}^{\beta}(f \circ g(x)) & =f(x)_{0}^{A} D_{x}^{\beta}(g(f(x))) \\
& =g(x)_{0}^{A} D_{x}^{\beta}(f(g(x))) .
\end{aligned}
$$

Definition 2: Let $f:[a, \infty) \rightarrow \mathbb{R}$ be the given function, then we propose that the beta-integral of $f$ is:

$$
{ }_{0}^{A} I_{x}^{\beta}(f(x))=\int_{0}^{x}\left(t+\frac{1}{\Gamma(\beta)}\right)^{\beta-1} f(t) d t .
$$

The above operator is the inverse operator of the proposed fractional derivative. We shall underpin this statement by the following theorem $[15,16]$.

Theorem 3: ${ }_{0}^{A} D_{x}^{\beta}\left[{ }_{0}^{A} I_{x}^{\beta}(f(x))\right]=f(x)$ for all $a \leq$ $x, \beta \in(0,1]$ with $f$ as a given continuous and differentiable function $[15,16]$.

\section{Derivation of the new groundwater flow equation}

To derive the new groundwater flow equation, we make use of the principle of continuity equation of flow, that is, the difference between the rate inflow and the rate outflow from annular cylinder is the equation of water volume change within the annular space. Thus:

$$
Q_{2}-Q_{1}={ }_{0}^{A} D_{t}^{\beta}[V(t)], 0<\beta \leq 1,
$$

where $Q_{1}$ is the rate of inflow, $Q_{2}$ is the rate of outflow, and the right hand side is the rate of volume change that account for the variability of the aquifer together with the locality of the flow within the annular space. The new definition of slope of the hydraulic gradient at the inner surface is $[21,22]$ :

$$
{ }_{0}^{A} D_{t}^{\beta}[V(t)]
$$

If $h$ is the height of piezometric surface above the impervious stratum, then the slope of hydraulic gradient line at the outer surface is provided as [22]:

$$
j=\frac{\partial h(r, t)}{\partial r}+\frac{\partial^{2} h(r, t)}{\partial r^{2}} .
$$

Nonetheless, using Darcy's law, we obtain:

$$
Q=K j \text { (Area of the flow). }
$$

Therefore, in the case of the inner flow, we have the following expression:

$$
Q_{1}=K\left(\frac{\partial h(r, t)}{\partial r}+\frac{\partial^{2} h(r, t)}{\partial r^{2}}\right)(2 \pi(r+d r) b) .
$$

In the case of outflow, we have the following expression:

$$
Q_{2}=\left(\frac{\partial h(r, t)}{\partial r}\right)(2 \pi r b)
$$

Here, $b$ is the thickness of the confined aquifer. Now from the definition of storage coefficient $(S), S$ is the 
volume of water released per unit surface area per unit change in head normal to the surface. Therefore, the change in volume is given as:

$$
\delta V=2 \pi r S d r d h .
$$

This leads us to the following equation [22]:

$$
{ }_{0}^{A} D_{t}^{\beta}[V(t)]=2 \pi r d r_{0}^{A} D_{t}^{\beta}[h(t)],
$$

where $t$ is the time since the beginning of pumping. However, substituting Eqs. (7) and (8) in Eq. (10), we obtain the following equation:

$$
\begin{aligned}
K\left(\frac{\partial h(r, t)}{\partial r}+\right. & \left.\frac{\partial^{2} h(r, t)}{\partial r^{2}}\right)(2 \pi(r+d r) b) \\
& -K\left(\frac{\partial h(r, t)}{\partial r}\right)(2 \pi r b) \\
& =2 \pi r S d r_{0}^{A} D_{0}^{\beta}(h(r, t))
\end{aligned}
$$

Now, dividing on both sides by $2 \pi r d r$ and neglecting the higher order terms, we obtain:

$$
\frac{\partial h(r, t)}{\partial r}+\frac{\partial^{2} h(r, t)}{\partial r^{2}}=\frac{S}{K b^{0}}{ }^{A} D_{0}^{\beta}(h(r, t)) .
$$

Thus, in terms of transmissivity, we have:

$$
\frac{\partial h(r, t)}{\partial r}+\frac{\partial^{2} h(r, t)}{\partial r^{2}}=\frac{S}{T^{A}}{ }_{0}^{\beta} D_{0}^{\beta}(h(r, t)) .
$$

The above equation is the modified groundwater equation of unsteady flow towards the well. In this equation, $h$ is hydraulic head, $r$ is radial distance from the well, $S$ is storage coefficient, $T$ is transmissivity, and $t$ is the time since the beginning of pumping [1-4,20-22].

\section{Derivation of analytical solution}

In this section, we present three different analytical methods for solving the new groundwater flow equation.

\subsection{Method of separation of variable}

Separation of variables, also known as the Fourier method, is any of the several methods for solving ordinary and partial differential equations, in which algebra allows one to rewrite an equation so that each of two variables occurs on a different side of the equation. For a partial differential equation with two parameters, the method assumes that the solution is in form of:

$$
h(r, t)=U(r) V(t) .
$$

Then, the above is replaced in the main equation, and two different equations are obtained with the inclusion of an eigenvalue. We shall use this method to derive the solution of the new groundwater flow equation. Now, replacing Eq. (13) into Eq. (12), we obtain:

$$
V(t) \frac{d U(r)}{d r}+V(t) \frac{d^{2} U(r)}{d r^{2}}=\frac{S_{T}^{A}}{T^{0}} D_{t}^{\beta}(V(t)) U(r) .
$$

Rearranging, we obtain the following equations:

$$
\left\{\begin{array}{l}
\frac{d U(r)}{d r}+\frac{d^{2} U(r)}{d r^{2}}=-\lambda^{2} U(r) \\
\frac{S}{T}{ }^{A} D_{t}^{\beta}[V(t)]=-\lambda^{2} V(t)
\end{array}\right.
$$

Here $\lambda$ is known as eigenvalues. The first equation in the above can be solved using the Sumudu transform. The Sumudu transform of a function posits that $f(x)$ is defined as:

$$
S(f(x))(u)=\int_{0}^{\infty} \frac{1}{u} \exp \left[-\frac{x}{u}\right] f(x) d x .
$$

Here are some useful properties of the Sumudu transform operator:

$$
\begin{aligned}
& S(f(x))(u)=\frac{F(u)-f(0)}{u}, \\
& S\left(f^{\prime \prime}(x)\right)(u)=\frac{F(u)-f(0)}{u^{2}}-\frac{f(0)}{u}, \\
& S\left[\frac{1}{x} f^{\prime}(x)\right](u)=\frac{1}{u} \frac{d F(u)}{d u} .
\end{aligned}
$$

Now applying on both sides of equation using the above properties, we obtain the following expression:

$$
\frac{1}{u} \frac{d U(u)}{d u}+\frac{U(u)-U(0)}{u^{2}}-\frac{U^{\prime}(0)}{u}=\lambda^{2} U(u) .
$$

Rearranging, and taking into account the boundary condition, we obtain:

$$
u \frac{d U(u)}{d u}+\left(1+(\lambda u)^{2}\right) U(u)=0 .
$$

Eq. (20) can further be arranged as follows:

$$
\frac{d U(u)}{U(u)}=\frac{(1+(\lambda u))^{2}}{u} d u .
$$

The above equation has an exact solution:

$$
U(u)=\exp \left[-\int \frac{\left(1+(\lambda u)^{2}\right)}{u}\right] .
$$

Nevertheless, applying the inverse Sumudu transform on both sides of Eq. (22), we obtain the following solution:

$$
U(r)=J_{0}\left(\lambda^{2} r\right)
$$


The above expression is known as the Bessel function of the first kind and is defined as:

$$
J_{0}[r]=\sum_{n=0}^{\infty} \frac{(-1)^{k}}{k !} \frac{1}{\Gamma(k+1)}\left(\frac{r}{2}\right)^{2 k} .
$$

The second equation of Relation (15) that is:

$$
\frac{S}{T^{A}}{ }^{0} D_{t}^{\beta}[V(t)]=-\lambda^{2} V(t)
$$

has as an exact solution:

$$
V(t)=\exp \left[-\frac{T \lambda^{2}}{\alpha S}\left(\left(t+\frac{1}{\Gamma(\beta)}\right)^{\beta}-\left(\frac{1}{\Gamma(\beta)}\right)^{\beta}\right)\right] .
$$

Thus, using the procedure of the separation of variables, we obtain the exact solution of the new groundwater flow equation as:

$$
\begin{aligned}
h(r, t)= & c \sum_{n-0}^{\infty} \exp \left[-\frac{T \lambda_{n}}{\alpha S}\left(\left(t+\frac{1}{\Gamma(\beta)}\right)^{\beta}\right.\right. \\
& \left.\left.-\left(\frac{1}{\Gamma(\beta)}\right)^{\beta}\right)\right] J_{0}\left[\lambda_{n} r\right] .
\end{aligned}
$$

Using the initial condition, we obtain the exact solution of the new groundwater equation to be:

$$
\begin{aligned}
h(r, t)= & \frac{Q}{4 \pi T} \sum_{n-0}^{\infty} \exp \left[-\frac{T \lambda_{n}}{\alpha S}\left(\left(t+\frac{1}{\Gamma(\beta)}\right)^{\beta}\right.\right. \\
& \left.\left.-\left(\frac{1}{\Gamma(\beta)}\right)^{\beta}\right)\right] J_{0}\left[\lambda_{n} r\right] .
\end{aligned}
$$

We shall now present an alternative way for solving this equation.

\subsection{Beta-Laplace transform method}

Due to the nature of the new groundwater equation, it is not possible for the common Laplace transform to be used in order to derive the exact solution from this equation. We shall, therefore, use the newly-introduced integral transform operator called the beta-Laplace transform or Atangana transform operator [16].

Definition 3: Let $f$ be a function such that for any $0<\beta \leq n$ the Laplace transform of $\left(t+\frac{1}{\Gamma(\beta)}\right)^{\beta-n} f(t)$ exists, then the Atangana-transform of $f$ is defined as:

$$
L_{\beta}(f(t))(s)=\int_{0}^{\infty}\left(t+\frac{1}{\Gamma(\beta)}\right)^{\beta-n} f(t) \exp [-s t] d t .
$$

The above operator has the following property:

$$
L_{\beta}\left[{ }_{0}^{A} D_{t}^{\beta}(f(t))\right]=s^{n} L(f(t))(s)-\sum_{k=1}^{n} s^{k-1} f^{(n-k)}(0) .
$$

Therefore, applying the Atangana-transform on both sides of Eq. (12), we obtain:

$$
\frac{\partial H(r, s)}{r \partial r}+\frac{\partial^{2} H(r, s)}{\partial r^{2}}=\frac{S}{T}(s H(r, s)-h(r, 0)) .
$$

We can now apply the Laplace transform on both sides to obtain:

$$
u \frac{d H(r, s)}{d u}+u^{2} H(r, s)-u^{2} H(0, s)-\lambda^{2} H(u, s)=0 .
$$

with:

$$
\lambda^{2}=s \frac{S}{T}
$$

Applying the boundary condition together with the initial condition, we obtain the following:

$$
u \frac{d H(u, s)}{d u}+\left(u^{2}-\lambda^{2}\right) H(u, s)=0 .
$$

The exact solution of the above equation is given as:

$$
U(r, s)=J_{0}\left(s \frac{S}{T} u\right)
$$

Therefore, applying the inverse Laplace twice for $s$ and $u$, we obtain the following exact solution:

$$
\begin{aligned}
h(r, t)= & c \int_{u}^{\infty} \frac{1}{t} \exp \left[-\frac{T \lambda^{2}}{\alpha S}\left(\left(t+\frac{1}{\Gamma(\beta)}\right)^{\beta}\right.\right. \\
& \left.\left.-\left(\frac{1}{\Gamma(\beta)}\right)^{\beta}\right)\right] d t,
\end{aligned}
$$

applying again the initial condition, we obtain the following exact solution of the new groundwater flowing within a confined aquifer:

$$
\begin{gathered}
h(r, t)=\frac{Q}{4 \pi T} \int_{u}^{\infty} \frac{1}{t} \exp \left[-\frac{T \lambda^{2}}{\alpha S}\left(\left(t+\frac{1}{\Gamma(\beta)}\right)^{\beta}\right.\right. \\
\left.\left.-\left(\frac{1}{\Gamma(\beta)}\right)^{\beta}\right)\right] d t=\frac{Q}{4 \pi T} W_{\beta}(u) \\
u=\frac{r^{2} S}{4 T t} h(r, t)=\frac{c}{t-t_{0}} \exp \left[-u_{\beta 0}\right] .
\end{gathered}
$$

The following integral will be referred to as betaexponential integral:

$$
\int_{0}^{\infty} \frac{1}{t} \exp \left[-\frac{T \lambda^{2}}{\alpha S}\left(\left(t+\frac{1}{\Gamma(\beta)}\right)^{\beta}-\left(\frac{1}{\Gamma(\beta)}\right)^{\beta}\right)\right] d t
$$




\subsection{Alternative method}

An alternative method is used here to derive the exact solution of the new groundwater flow equation. This method is often used for some classes of parabolic partial differential equation. This method used the concept of reduction of dimension; in particular, the method used the Boltzmann transformation. In this method, they defined an arbitrary $t_{0}<t$ by the equation:

$$
u_{0}=\frac{S r^{2}}{4 T\left(t-t_{0}\right)} .
$$

However, in the case of the new groundwater flow equation, the above equation cannot be used, and to extend this method to the scope of beta-partial differential equation, we propose the following transformation:

$$
u_{\beta 0}=\frac{S r^{2}}{4 T\left[\left(t-t_{0}+\frac{1}{\Gamma(\beta)}\right)-\left(\frac{1}{\Gamma(\beta))} \beta\right]\right.} .
$$

Now, let us consider the following function:

$$
h(r, t)=\frac{c}{t-t_{0}} \exp \left[-u_{\beta 0}\right]
$$

with $c$ being any arbitrary constant. If we assume that $r_{b}$ is the ratio of the borehole from which the water is being taken out from the aquifer, thus, the total volume of the water withdrawn from the aquifer is provided by:

$$
Q_{0} \Delta t_{0}=4 \pi c T \text {. }
$$

Here:

$$
h(r, t)=\frac{c}{t-t_{0}} \exp \left[-u_{\beta 0}\right],
$$

is the drawdown which will be experimental at a detachment, $r$ from the pumping well after the time space of $\Delta t_{0}$. Now, $\gamma$, assume that the above formula is continual $m$-times, meaning that water is being removed for a very short period of time, $\Delta t_{k}$, at consecutive times. $t_{k+1}=t_{k}+\Delta t_{k},(k=0,1,2, \ldots, m)$. In this instance, since the new groundwater flow equation is linear, the total drawdown at any time $t>t_{k}$ is given by:

$$
h(r, t)=\frac{1}{4 \pi T} \sum_{k=0}^{n} \frac{\Delta t_{k} Q_{k}}{t-t_{k}} \exp \left[-u_{\beta 0}\right] .
$$

Note that in the above equation, the summation can be transformed into an integral if $\Delta t \rightarrow 0$, Then Eq. (37) becomes:

$$
h(r, t)=\frac{1}{4 \pi T} \int_{t_{0}}^{t} Q(x) \frac{\exp \left[-u_{\beta 0}\right]}{t-x} d x .
$$

A particularly important solution which arises when $t_{0}$ is considered at the origin zero and at the point the discharge rate is independent of time, then Eq. (36) becomes:

$$
\begin{aligned}
h(r, t)= & \frac{Q}{4 \pi T} \int_{u}^{\infty} \frac{1}{t} \exp \left[-\frac{T \lambda^{2}}{\alpha S}\left(\left(t+\frac{1}{\Gamma(\beta)}\right)^{\beta}\right.\right. \\
& \left.\left.-\left(\frac{1}{\Gamma(\beta)}\right)^{\beta}\right)\right] d t=\frac{Q}{4 \pi T} W_{\beta}(u) .
\end{aligned}
$$

\section{Numerical simulations}

In this section, we shall show some numerical simulations of the new groundwater flow model; here we use $Q=100, T=10$, and $S=10^{-5}$. The plots are shown for two different values of $\alpha$. The simulations are depicted in Figures 1 and 2 as the time-space change of water level. Figures 3 and 4 show the contour plot as the function of time and space.

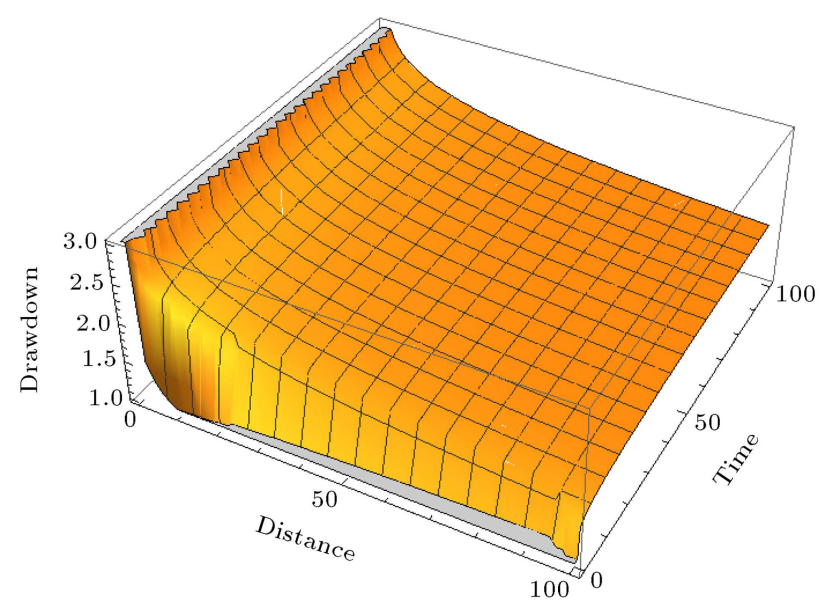

Figure 1. Exact solution of the new groundwater flow equation as a function of $x$ and $t$ for $\alpha=0.6$.

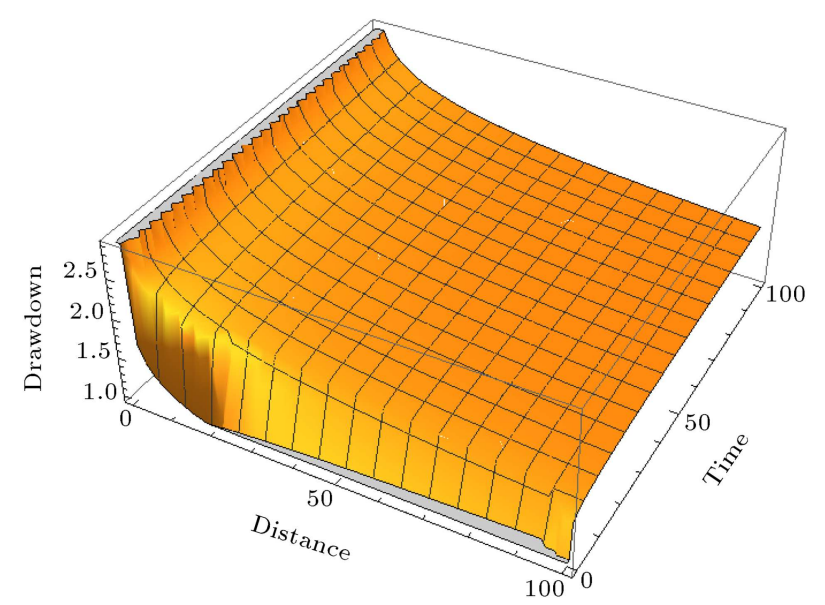

Figure 2. Exact solution of the new groundwater flow equation as a function of $x$ and $t$ for $\alpha=0.5$. 


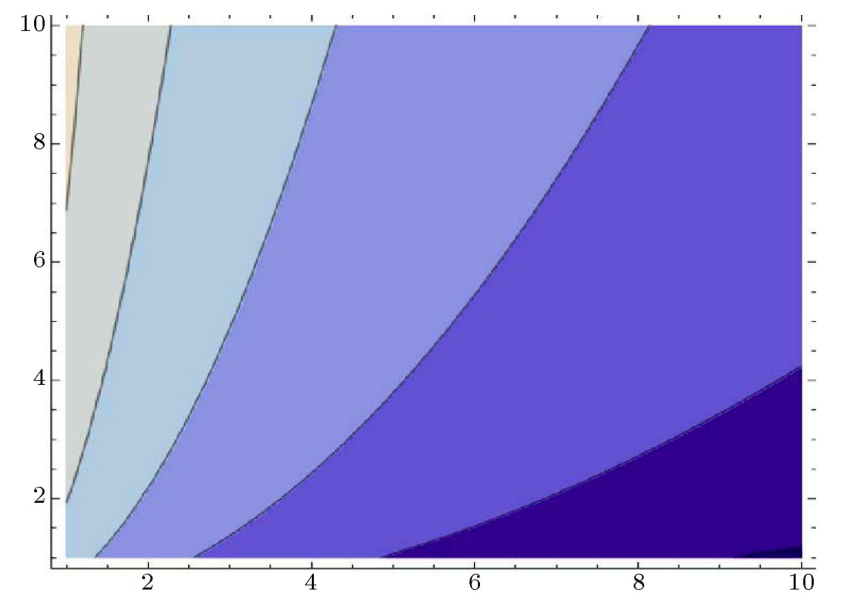

Figure 3. Contour plot exact solution of the new groundwater flow equation as a function of $x$ and $t$ for $\alpha=0.6$.

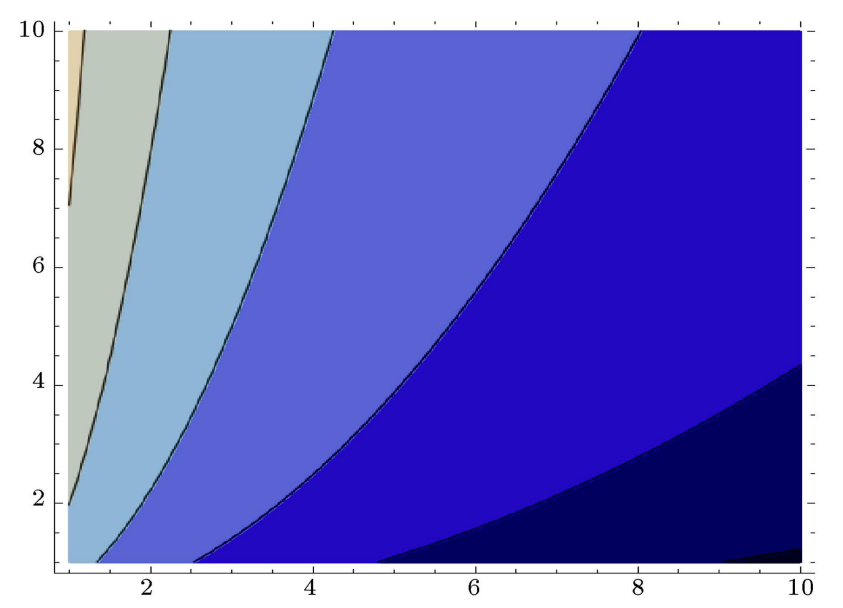

Figure 4. Contour plot of exact solution of the new groundwater flow equation as a function of $x$ and $t$ for $\alpha=0.5$.

\section{Conclusion and discussion}

Groundwater is an important source of potable water; consequently, many studies are required to accurately understand the movement of this water within the geological formation called aquifer. This description is usually achieved using mathematical formula, for instance, the Theis groundwater flow equation. However, this equation uses the concept of derivative. The Theis equation uses the Newton version of derivative that does not accurately describe many physical problems as were revealed in many researches in the literature. The modified equation by Botha and Cloot used the Riemann-Liouville fractional derivative. This version does describe the flow non-locally, therefore, it cannot be used at the borehole. In addition, it uses nonconventional initial and boundary conditions. A modified one by Atangana, using the Caputo derivative, also describes the flow non-locally. Although it uses the usual initial and boundary conditions, it cannot be used at the borehole. This new model describes the flow locally and also describes it globally.

\section{References}

1. Theis, C.V. "The relation between the lowering of the piezometric surface and the rate and duration of discharge of well using groundwater storage", Am. Geophys. Union Trans., 16, pp. 519-524 (1935).

2. Hantush, M.S. "Flow of ground water in sands of non-uniform thickness. Part 3. Flow to wells", Jour. Geophys. Res., 67(4), pp. 1527-1534 (1962).

3. Cooper, H.H. and Jacob. C.E. "A generalized graphical method for evaluating formation constants and summarizing well field history", Am. Geophys. Union Trans., 27, pp. 526-534 (1946).

4. Novakowski, K.S. "A composite analytical model for analysis of pumping tests affected by well bore storage and finite thickness skin", Water Resources Research, 25(9), pp. 1937-1946 (1989).

5. Caputo, M. "Linear models of dissipation whose $\mathrm{Q}$ is almost frequency independent-part II", Geophysical Journal International, 13(5), pp. 529-539 (1967).

6. Podlubny, I. "Geometric and physical interpretation of fractional integration and fractional differentiation", Fractional Calculus \& Applied Analysis, 5(4), pp. 367386 (2002).

7. Benson, D.A., Wheatcraft, S.W. and Meerschaert, M.M. "Application of a fractional advection-dispersion equation", Water Resources Research, 36(6), pp. 14031412 (2000).

8. Wheatcraft, S.W. and Tyler, S.W. "An explanation of scale-dependent dispersivity in heterogeneous aquifers using concepts of fractal geometry", Water Resources Research, 24(4), pp. 566-578 (1988).

9. Kilbas, A.A., Srivastava, H.M. and Trujillo, J.J., Theory and Applications of Fractional Differential Equations, 204, Elsevier Science B.V., Amsterdam, The Netherlands (2006).

10. Cloot, A. and Botha, J.F. "A generalised groundwater flow equation using the concept of non-integer order derivatives", Water $S A, 32(1)$, pp. 55-78 (2006).

11. Atangana, A. and Vermeulen, P.D. "Analytical solutions of a space-time fractional derivative of groundwater flow equation", Abstract and Applied Analysis, Article ID 381753, pp. 1-11 (2014).

12. Atangana, A. and Bildik, N. "The use of fractional order derivative to predict the groundwater flow", Mathematical Problems in Engineering, Article ID 543026, pp. 1-9 (2013).

13. Atangana, A. and Botha, J.F. "Generalized groundwater flow equation using the concept of variable order derivative", Boundary Value Problems, Article 53 (2013).

14. Atangana, A. and Doungmo Goufo, E.F. "On the 
mathematical analysis of Ebola hemorrhagic fever: Deathly infection disease in west african countries", BioMed Research International, Article ID 261383, pp. 1-7 (2014).

15. Atangana, A. and Doungmo Goufo, E.F. "Extension of matched asymptotic method to fractional boundary layers problems", Mathematical Problems in Engineering, Article ID 107535, pp. 1-7 (2014).

16. Atangana, A. "A novel model for the Lassa haemorrhagic fever: Deathly disease for pregnant women", Neutral Computing and Application, 26(8), pp. 18951903 (November 2015).

17. Yang, X.J. and Srivastava, H.M. "An asymptotic perturbation solution for a linear oscillator of free damped vibrations in fractal medium described by local fractional derivatives", Communications in Nonlinear Science and Numerical Simulation, 29(1), pp. 499-504 (2015).

18. Yang, X.J., Srivastava, H.M., He, J.H. and Baleanu, D. "Cantor-type cylindrical-coordinate method for differential equations with local fractional derivatives", Physics Letters A, 377(28), pp. 1696-1700 (2013).

19. Yang, X.J., Baleanu, D. and Srivastava, H.M. "Local fractional similarity solution for the diffusion equation defined on Cantor sets". Applied Mathematics Letters, 47, pp. 54-60 (2015).

20. Atangana, A. and Botha, J.F. "Analytical solution of the groundwater flow equation obtained via homotopy decomposition method", J Earth Sci Climate Change, 3(115) (2012). Doi: 10.4172/2157-7617.1000115

21. Atangana, A. and Doungmo Goufo, E.F. "A model of the groundwater flowing within a leaky aquifer using the concept of local variable order derivative", J. Nonlinear Sci. Appl., 8(6), pp. 763-775 (2015).

22. Groundwater flow to wells, Chapter 3. http://www.hwe.org.ps/Education/Birzeit/GroundwaterEngineering/Chapter $\% 203 \% 20-\%$

\section{Biographies}

Abdon Atangana obtained his BS degree in pure mathematics, his Honors degree and MS degree in Applied Mathematics with distinction from University of Free State, South Africa. He also obtained his $\mathrm{PhD}$ degree in applied mathematics at the Institute for Groundwater Studies, University of the Free State, Bloemfontein, South Africa. He is serving as editor of some international journals, and also reviewer of more than 100 international accredited journals. He has been the Lead and Guest Editor of some special issues, and has presented and participated in more than 15 international conferences. His research interests are methods and applications of partial and ordinary differential equations, fractional differential equations, perturbations methods, asymptotic methods, iterative methods, and groundwater modeling. He is the author of the book "Derivative with new parameter: theory, methods and applications," published by Academic Press Elsevier.

Canan Ünlü obtained her Master and $\mathrm{PhD}$ degree at the Istanbul University, Turkey. She is an Assistant Researcher at the Istanbul University. She has participated in more than 10 international conferences. Currently she is working on fractional differential equations and their applications. 